\title{
Discrete Complex Functions With Prescribed Boundary Values and Residues*
}

\author{
Elmor L. Peterson **
}

\begin{abstract}
(March 9, 1967)
R. Isaacs, J. Ferrand, R. J. Duffin, and several others have developed a function theory for "discrete analytic functions" defined on "discrete regions" in the "discrete complex plane." In this paper we bring to light some combinatorial-topological properties of "simple discrete regions," and we study some basic properties of discrete analytic functions that are defined on simple discrete regions. These combinatorial-topological properties and basic properties are then used to establish an existence and uniqueness theorem for discrete complex functions with prescribed "boundary values" and "residues" on an arbitrary simple discrete region.
\end{abstract}

Key Words: Analytic functions, complex analysis, Dirichlet problem, discrete analytic functions.

\section{Introduction}

This paper is concerned with complex functions defined at the nodes of a square mesh $\mathscr{M}_{h}$ that lies in the complex plane and is depicted in figure 1.1. The theory of these functions was initiated by R. Isaacs $[1]^{1}$ and J. Ferrand [2] and has been extensively developed by R. J. Duffin [3] and several of his students $[4,5,6,7]$. The functions investigated are said to be "discrete analytic" and are discrete analogs of analytic functions of a continuous complex variable. A complex function $f$ is discrete analytic at a square $S$ belonging to $\mathscr{M}_{h}$ if the difference quotient of $f$ across one diagonal of $S$ is equal to the difference quotient of $f$ across the other diagonal of $S$.

The results obtained in [1,2, and 3] include discrete analogs of the Cauchy Riemann equations, Laplace's equation, the maximum principle, conjugate harmonic functions, differentiation, integration, the residue theorem, Cauchy's theorem, Morera's theorem, Cauchy's integral formula, and polynomials. Some of the concepts studied have no direct analogy in the classical continuous theory. These include the notions of duality and bipolynomials.

In [3] it is shown that for each real function $u$ that is "discrete harmonic" on a "simple discrete region $\mathscr{R}$ " there is a real "conjugate function $v$ ' that is discrete harmonic on $\mathscr{R}$ such that $u+i v$ is discrete analytic on $\mathscr{R}$. This theorem becomes a corollary to the main theorem of this paper, which is stated and proved in section 3 as Theorem 3.5. The main theorem

\footnotetext{
*An invited paper. Written while the author was visiting the mathematics department at West Virginia University and based on part of the author's Ph. D. thesis, which was written under the guidance of Professor R. J. Duffin at Carnegie Institute of Technology with financial support from a Socony Mobil Fellowship and Research Grant DA-AROD. 31-124-G78 from the U. S. Army Research Office-Durham. The author wishes to thank A. J. Goldman (NBS) for his many helpful suggestions on improving this paper.

***Present address: Department of Mathematics, University of Michigan, Ann Arbor, 48104 .

'Figures in brackets indicate the literature references at the end of this paper.
}

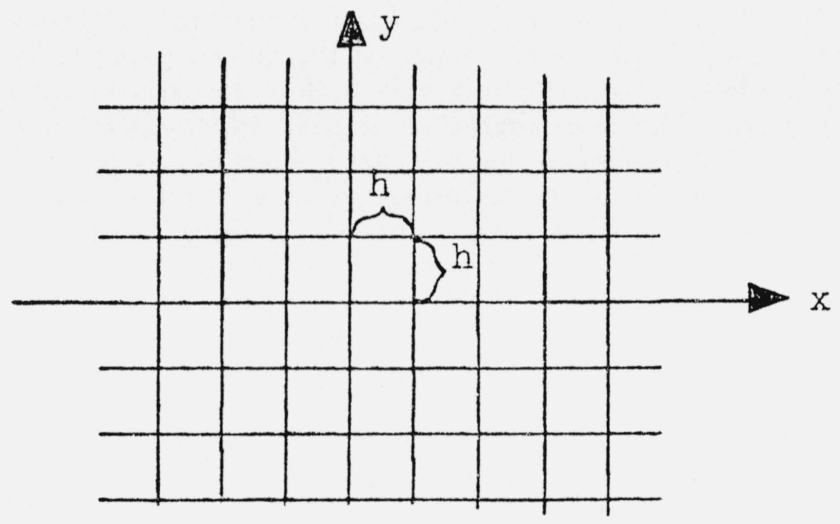

Figure 1.1. The discrete complex plane.

discusses both the existence and the uniqueness of a function $u+i v$ that has prescribed "residue" and "boundary values" on a simple discrete region $\mathscr{R}$.

The proof of the main theorem depends on some properties of simple discrete regions and on some basic properties of discrete functions. The required properties of simple discrete regions are stated and proved in section 2 , and the required basic properties of discrete functions are stated and proved in the first part of section 3. With the possible exception of Euler's formula [8] this paper is completely self-contained.

\section{The Discrete Complex Plane and Simple Discrete Regions}

The mesh $\mathscr{M}_{h}$, as shown in figure 1.1, consists of a union of nonoverlapping squares, each of whose sides has length $h$. The lattice $\mathscr{L}_{h}$ (or discrete complex plane) is composed of the points that fall on a vertex 
of a member of $\mathscr{M}_{h}$. Each member $z$ of $\mathscr{L}_{h}$ can be represented as $z=x h+i y h$ where $x$ and $y$ are appropriate integers. The even lattice $\mathscr{E}_{h}$ consists of those lattice points for which $x+y$ is even, and the odd lattice $\mathscr{O}_{h}$ consists of those lattice points for which $x+y$ is odd.

A chain is an oriented set of lattice points $z_{0}$, $z_{1}, \ldots . ., z_{n}$ with the property that the straight line joining $z_{i-1}$ to $z_{i}$ lies along an edge of a square belonging to $\mathscr{M}_{h}$. A chain $z_{0}, z_{1}, \ldots, z_{n}$ for which $z_{0}=z_{n}$ is called a simple closed chain when its elements, exclusive of $z_{0}$ and $z_{n}$, are distinct. An even chain (odd chain) is an oriented set of even (odd) lattice points $z_{0}, z_{1}, \ldots ., z_{n}$ with the property that the straight line joining $z_{i-1}$ to $z_{i}$ lies along a diagonal of a square belonging to $\mathscr{M}_{n}$.

A set of lattice points is said to be connected if each pair of points belonging to the set can be connected by a chain so that each point of the chain lies in the set. A set of lattice points is said to be evenly connected (oddly connected), if each pair of even (odd) lattice points belonging to the set can be connected by an even (odd) chain so that each point of the even (odd) chain lies in the set.

A discrete region in the discrete complex plane $\mathscr{L}_{h}$ consists of the nodes of a union of squares from $\mathscr{M}_{h}$. The discrete region is said to be constructed from these squares, and the union of the closed planar sets bounded by these squares is called the associated region. The boundary of a discrete region consists of those lattice points that belong to the discrete region and lie on the boundary of its associated region. The union of all lattice points that belong to the discrete region but do not belong to its boundary is called the interior of the discrete region. Lemma 2.1 is an obvious consequence of these definitions.

LEMMA 2.1: A lattice point $\mathrm{z}$ is an interior point of a discrete region $\mathscr{R}$ if, and only if, each of the four squares having $\mathrm{z}$ as a vertex belongs to the family of squares used in the construction of $\mathscr{R}$.

A discrete region is said to be finite if it consists of a finite number of lattice points. A simple discrete region is a finite discrete region whose boundary can be represented by a simple closed chain.

We shall now deduce some useful properties of simple discrete regions. It does not seem worthwhile to detail the combinatorial-topological machinery required for full formal rigor in the demonstration of these intuitively evident properties; instead, we aim at leaving the reader with a clear picture of just what "sub-lemmas" would have to be verified when using such machinery in a fully detailed treatment.

Most of the proofs rely on induction. The following lemma provides the key to these proofs.

LEMMA 2.2: If $\mathscr{R}_{s}$ is a simple discrete region constructed from s squares and $\mathrm{s} \geqslant 2$, then $\mathscr{R}_{\mathrm{s}}$ is the union of a simple discrete region $\mathscr{R}_{\mathrm{s}-1}$ constructed from $\mathrm{s}-1$ squares and a simple discrete region $\mathscr{R}_{1}$ constructed from one square.

Proof: Let $C: z_{0}, z_{1}, \ldots ., z_{n}$ be the simple closed chain that represents the boundary of $\mathscr{R}_{s}$, and suppose that $C$ is oriented so that $\mathscr{R}_{s}$ lies to the left as $C$ is traversed from $z_{0}$ to $z_{n}=z_{0}$. Since $C$ is finite and closed, there exists a lattice point $z_{t} \epsilon C: z_{0}, \ldots, z_{t}$,
. . ., $z_{n}$ at which $C$ makes a left turn. The lattice can clearly be rotated so that $z_{t-1}, z_{t}$, and $z_{t+1}$ have the positions shown in figure 2.1. According to our choice of $C$ the square $S_{1}$ is contained in the associated region $R_{s}$ of $\mathscr{R}_{s}$. Since $s \geqslant 2$, at least one of the squares $S_{2}, S_{3}$, and $S_{4}$ must be contained in $R_{s}$. We must distinguish seven cases.

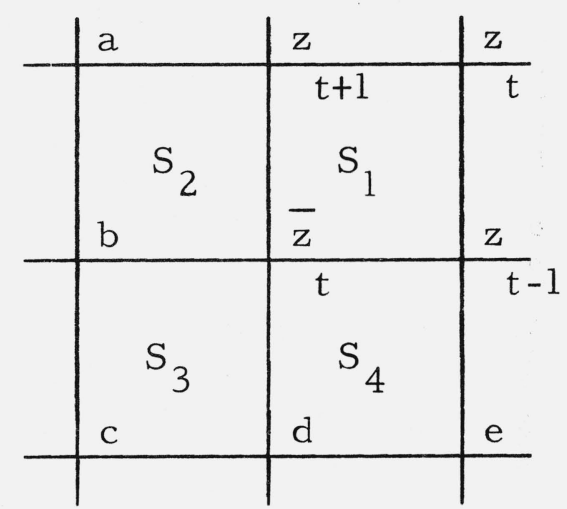

FigURE 2.1. A left turn in a simple closed chain.

CASE I: $S_{2}, S_{3}$, and $S_{4}$ are contained in $R_{s}$. Then consider the closed chain $\bar{C}: z_{0}, . . ., z_{t-1}, z_{t}, z_{t+1}$, ..., $z_{n}$. Since $\bar{z}_{t}$ is an interior point of $\mathscr{R}_{s}, \bar{C}$ is simple and hence encloses a simple discrete region $\mathscr{R}_{s-1}$ $\subset \mathscr{R}_{s}$. In this case $R_{1}=S_{1}$.

CASE II: $S_{2}$ and $S_{3}$ are contained in $R_{s}$ but $S_{4}$ is not. This means that $\overline{z_{t}}$ precedes $z_{t-1}$ in $C$ (i.e., $C: z_{0}, \ldots$., $\left.\bar{z}_{t}, z_{t-1}, z_{t}, z_{t+1}, \ldots, z_{n}\right)$. Now let $\bar{C}: z_{0}, \ldots, \bar{z}_{t}$, $z_{t+1}, \ldots, z_{n}$ be obtained from $C$ by deleting $z_{t-1}$ and $z_{t}$. Then ' $\bar{C}$ is clearly simple and encloses a simple discrete region $\mathscr{R}_{s-1} \subset \mathscr{R}_{s}$. In this case $R_{1}=S_{1}$.

CASE III: $S_{2}$ and $S_{4}$ are contained in $R_{s}$ but $S_{3}$ is not. This means that the sequence $b, \bar{z}_{t}, d$ appears in $C$ and hence $C$ makes a right turn at $\overline{z_{t}}$. Since there must be four more left turns than right turns in $C$, we could have initially chosen $z_{t}$ so as to eliminate this case.

CASE IV: $S_{3}$ and $S_{4}$ are contained in $R_{s}$ but $S_{2}$ is not. This case is handled in a manner similar to Case II.

CASE V: $S_{2}$ is contained in $R_{s}$, but $S_{3}$ and $S_{4}$ are not. This means that the sequence $b, \overline{z_{t}}, z_{t-1}, z_{t}, z_{t+1}$ belongs to $C$ (i.e., $C: z_{0}$. . ., $b, \bar{z}_{t}, z_{t-1}, z_{t}, z_{t+1}, \ldots ., z_{n}$ ). Now let $\bar{C}: z_{0}, \ldots ., b, \bar{z}_{t}, z_{t+1}, \ldots ., z_{n}$ be obtained from $C$ by deleting $z_{t-1}$ and $z_{t}$. Then $\bar{C}$ is clearly simple and encloses a simple discrete region $\mathscr{R}_{s-}$ $\subset \mathscr{R}_{s}$. In this case $R_{1}=S_{1}$.

CASE VI: $S_{3}$ is contained in $R_{s}$ but $S_{2}$ and $S_{4}$ are not. This case cannot occur, since $\overline{z_{t}}$ would have to appear twice in $C$, which would mean that $C$ is not simple.

CASE VII: $S_{4}$ is contained in $R_{s}$, but $S_{2}$ and $S_{3}$ are not. This case is handled in a manner similar to Case V.

All possibilities have been exhausted, so the proof of Lemma 2.2 is complete.

We can now derive some useful properties of simple discrete regions.

LEMMA 2.3: If $\mathscr{R}_{\mathrm{S}}$ is a simple discrete region constructed from $\mathrm{s}$ squares and $\mathrm{s} \geqslant 1$, then $\mathscr{R}_{\mathrm{s}}$ is connected and both evenly and oddly connected. 
Proof: The theorem is obviously true for $s=1$. If $s>1$, then Lemma 2.2. shows that $\mathscr{R}_{s}=\mathscr{R}_{s-1} \cup \mathscr{R}_{1}$. Using induction, we assume that both $\mathscr{R}_{s-1}$ and $\mathscr{R}_{1}$ have the desired properties. Since $\mathscr{R}_{s}$ is simple, $\mathscr{R}_{s-1}$ and $\mathscr{R}_{1}$ must have at least one even lattice point in common and at least one odd lattice point in common. The rest of the proof is trivial.

LEMMA 2.4: If $\mathscr{R}_{\mathrm{S}}$ is a simple discrete region constructed from $\mathrm{s}$ squares and $\mathrm{S}_{1}$ is one of the squares used in constructing $\mathscr{R}_{\mathrm{s}}$, then $\mathrm{S}_{1}$ is the first member of a sequence $\left\{\mathrm{S}_{\mathrm{j}}\right\}_{\mathrm{j}=1}^{\mathrm{s}}$ of squares belonging to $\mathscr{M}_{\mathrm{h}}$ such that

(1) $\mathscr{R}_{\mathrm{s}}$ is constructed from $\bigcup_{\mathrm{j}=1}^{\mathrm{s}} \mathrm{S}_{\mathrm{j}}$, and

(2) For each $\mathrm{j}$ such that $1<\mathrm{j} \leqslant \mathrm{s}$, there is an integer $\mathrm{k}$ such that $\mathrm{l} \leqslant \mathrm{k}<\mathrm{j}$ and such that $\mathrm{S}_{\mathrm{j}}$ has a side in common with $\mathrm{S}_{\mathrm{k}}$.

Proof: The proof, being trivial for $s=1$ and $s=2$, is accomplished through induction on $s$. If $s \geqslant 2$, then Lemma 2.2 shows that $\mathscr{R}_{s}=\mathscr{R}_{s-1} \cup \mathscr{R}_{1}$. Two cases must be treated.

CASE I: $S_{1}$ is used in the construction of $\mathscr{R}_{s-1}$. Then there exists a sequence $\left\{S_{j}\right\}_{j=1}^{s-1}$ that satisfies condition (2) and is used for the construction of $\mathscr{R}_{s-1}$. Letting $S_{s}$ be the square used in constructing $\mathscr{R}_{1}$ and remembering that the boundary of $\mathscr{R}_{s}$ is simple, we see that $S_{s}$ must have a side in common with a member of $\left\{S_{j}\right\}_{j=1}^{s-1}$, which means that $\left\{S_{j}\right\}_{j=1}^{s}$ satisfies both condition (1) and condition (2).

CASE II: $S_{1}$ is used in the construction of $\mathscr{R}_{1}$. Since the boundary of $\mathscr{R}_{s}$ is simple, we can choose a square $S_{2}$ that is used in the construction of $\mathscr{R}_{s-1}$ and has a side in common with $S_{1}$. Then there is a sequence $\left\{S_{j+1}\right\}_{j=1}^{s-1}$ that satisfies condition (2) and is used for the construction of $\mathscr{R}_{s-1}$. It is now easily seen that the sequence $\left\{S_{j}\right\}_{j=1}^{s}$ satisfies conditions (1) and (2). This completes the proof of Lemma 2.4.

LEMMA 2.5: Let $\mathrm{p}$ be the number of lattice points in a simple discrete region $\mathscr{R}_{\mathrm{S}}$ that is constructed from $\mathrm{s}$ squares and has $\mathrm{b}$ boundary points. Then $2 \mathrm{p}-\mathrm{b}-2=2 \mathrm{~s}$.

Proof: The proof of this lemma can be accomplished through induction on $s$. A shorter proof can be based on Euler's formula from the theory of planar graphs [8]. If a planar graph is connected, the Euler formula states that

$$
v-e+f=2,
$$

where $v$ is the number of vertices in the graph, $e$ is the number of edges in the graph, and $f$ is the number of regions into which the plane is divided by the graph. In order to apply this formula we must consider the connected planar graph $G$ that results from taking the union of the squares used in the construction of $\mathscr{R}_{s}$. The number of vertices in $G$ is simply the number of lattice points in $\mathscr{R}_{s}$, that is,

$$
v=p .
$$

Since $s$ squares are used in constructing $G$, the number of regions into which the plane is divided is $s+1$; thus

$$
f=s+1 \text {. }
$$

There are $b$ edges connecting the boundary points of $\mathscr{R}_{s}$, because the boundary of $\mathscr{R}_{s}$ is a simple closed chain. Each of these edges borders only one of the squares used in the construction of $\mathscr{R}_{s}$. All other edges belonging to $G$ border two of the squares used in the construction of $\mathscr{R}_{s}$. Since each square is bordered by 4 edges, it follows quite easily that

$$
2 e=4 s+b
$$

Multiplying eq (2.1) by 2 and substituting eqs (2.2), (2.3), and (2.4) into the result proves Lemma 2.5.

Figure 2.2 shows the union of ten squares to form a simple discrete region $\mathscr{R}_{10}$. Odd lattice points are denoted by crosses, and even lattice points are represented by dots. The square labeled with $S_{j}$ occupies the $j$ th position in the ordered sequence $\left\{S_{j}\right\}_{j=1}^{10}$ of squares used to construct $\mathscr{R}_{10}$. The points $z_{1}, z_{2}, z_{3}$, and $z_{4}$ form a chain. An even chain $z_{1}^{e}, z_{2}^{e}, z_{3}^{e}, z_{4}^{e}$ is shown along with an odd chain $z_{1}^{0}, z_{2}^{0}, z_{3}^{0}$. The boundary of $\mathscr{R}_{10}$, denoted by $\partial \mathscr{R}_{10}$, is depicted by the simple closed chain $\bar{z}_{1}, \bar{z}_{2}, \ldots ., \bar{z}_{16}$. The node $z_{i}$ is an example of an interior point of $\mathscr{R}_{10}$.

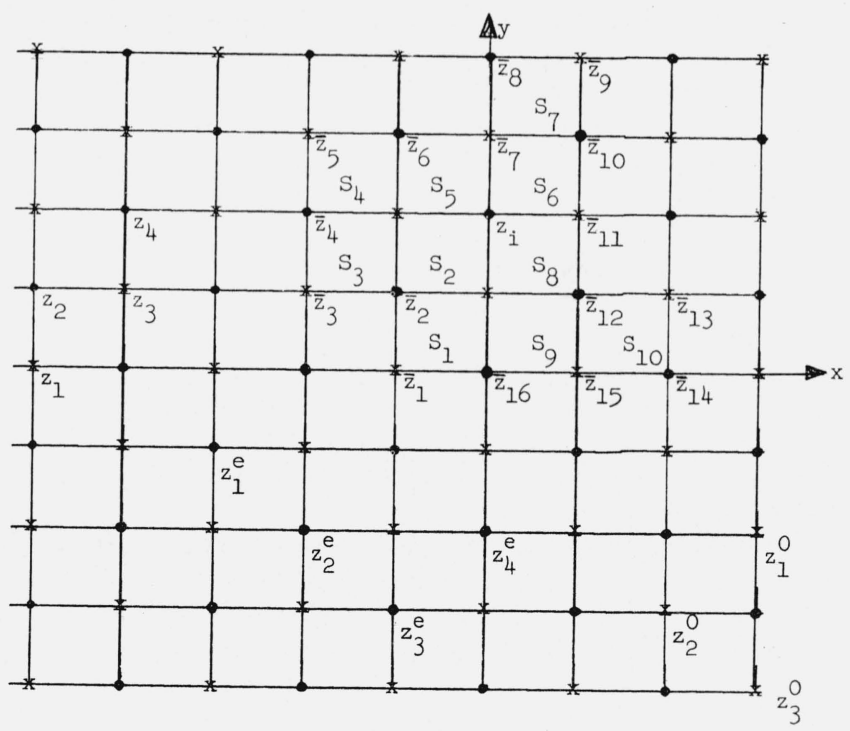

Figure 2.2. Chains and a simple discrete region.

\section{The Main Theorem}

We begin by defining a finite difference operator $L$ whose definition is taken from [3].

DEF. 3.1: Let $\mathrm{f}$ be a complex lattice function and suppose that $\mathrm{S}$ is a square belonging to $\mathscr{M}_{\mathrm{h}}$. Then the residue $\mathrm{L}(\mathrm{f}, \mathrm{S})$ of $\mathrm{f}$ at the square $\mathrm{S}$ is defined by

$$
\mathrm{L}(\mathrm{f}, \mathrm{S})=\mathrm{f}\left(\mathrm{z}_{0}\right)+\operatorname{if}\left(\mathrm{z}_{1}\right)+\mathrm{i}^{2} \mathrm{f}\left(\mathrm{z}_{2}\right)+\mathrm{i}^{3} \mathrm{f}\left(\mathrm{z}_{3}\right),
$$


where $\mathrm{z}_{0}, \mathrm{z}_{1}, \mathrm{z}_{2}$, and $\mathrm{z}_{3}$ have the orientation depicted in figure 3.1 and $\mathrm{i}=\mathrm{V}-1$.

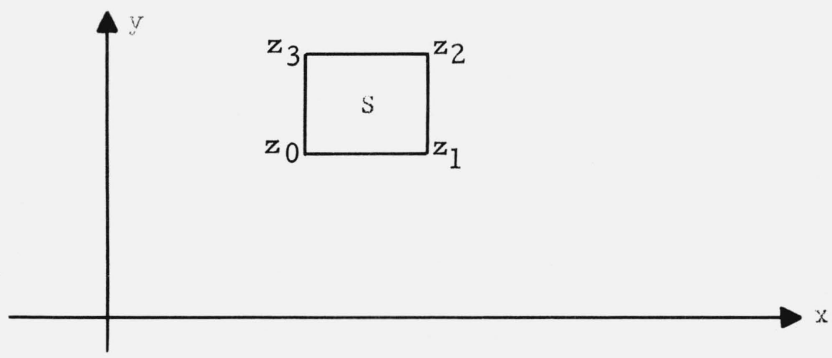

Figure 3.1. Orientation of the square $\mathrm{S}$.

The quantity $L(f, S)$ is termed the residue of $f$ at $S$, because in "discrete contour integration" it plays a role [3] analogous to the role played by the residue of a complex function of a continuous complex variable in the classical theory of contour integration.

DEF. 3.2: A lattice function $\mathrm{f}$ is discrete analytic at a square $\mathrm{S}$ belonging to $\mathscr{M}_{\mathrm{h}}$ if its residue $\mathrm{L}(\mathrm{f}, \mathrm{S})$ at $\mathrm{S}$ is zero. A lattice function $\mathrm{f}$ is discrete analytic in a discrete region $\mathscr{R}$ if it is discrete analytic at each square used in the construction of $\mathscr{R}$. The reader should have no trouble showing that this definition of discrete analyticity is equivalent to the one given in the first paragraph of section 1 .

To find analogues of the Cauchy Riemann equations, one need only set the real and imaginary parts of $L(f, S)$ equal to zero. This gives the following theorem, which is taken from [3].

THEOREM 3.1: Let $\mathrm{f}=\mathrm{u}+\mathrm{iv}$ where $\mathrm{u}$ and $\mathrm{v}$ are real lattice functions. Then $\mathrm{f}$ is discrete analytic at $\mathrm{S} \epsilon \mathscr{M}_{\mathrm{h}}$ if and only if

$$
\mathrm{u}\left(\mathrm{z}_{2}\right)-\mathrm{u}\left(\mathrm{z}_{0}\right)=\mathrm{v}\left(\mathrm{z}_{3}\right)-\mathrm{v}\left(\mathrm{z}_{1}\right)
$$

and

$$
\mathrm{v}\left(\mathrm{z}_{2}\right)-\mathrm{v}\left(\mathrm{z}_{0}\right)=-\left[\mathrm{u}\left(\mathrm{z}_{3}\right)-u\left(z_{1}\right)\right],
$$

where $\mathrm{z}_{0}, \mathrm{z}_{1}, \mathrm{z}_{2}$, and $\mathrm{z}_{3}$ have the orientation shown by square $\mathrm{S}$ of figure 3.1.

Following the example of the theory of functions of a continuous complex variable, we give a definition of discrete harmonicity. This definition, which is taken from [3], is justified by Lemma 2.1.

DEF. 3.3: Suppose $\mathrm{f}$ is a complex lattice function defined on a discrete region $\mathscr{R}$ of the discrete complex plane $\mathscr{L}_{\mathrm{h}}$. Let a function $\mathrm{H}(\mathrm{f}, \cdot)$, which maps the interior points of $\mathscr{R}$ into the field of complex numbers, be defined by

$$
\mathrm{H}\left(\mathrm{f}, \mathrm{z}_{\mathrm{i}}\right) \equiv \mathrm{f}\left(\mathrm{z}_{1}\right)+\mathrm{f}\left(\mathrm{z}_{2}\right)+\mathrm{f}\left(\mathrm{z}_{3}\right)+\mathrm{f}\left(\mathrm{z}_{4}\right)-4 \mathrm{f}\left(\mathrm{z}_{\mathrm{i}}\right)
$$

where $\mathrm{z}_{\mathrm{i}}, \mathrm{z}_{1}, \mathrm{z}_{2}, \mathrm{z}_{3}$, and $\mathrm{z}_{4}$ have the positions shown in figure 3.2. Then $\mathrm{f}$ is said to be discrete harmonic at an interior point $\mathrm{z}_{\mathrm{i}}$ of $\mathscr{R}$ if $\mathrm{H}\left(\mathrm{f}, \mathrm{z}_{\mathrm{i}}\right)=0$.

A simple consequence of this definition is the "maximum principle" given by Theorem 3.2.

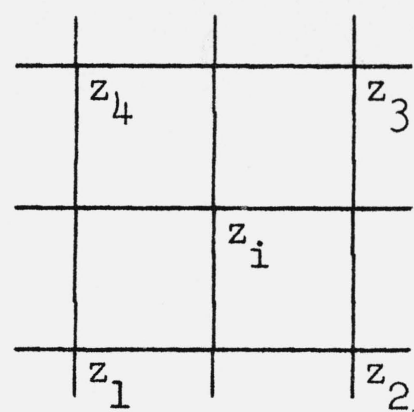

FiguRE 3.2. An interior point.

THEOREM 3.2: If $\mathrm{f}$ is a complex lattice function that is discrete harmonic at each interior point of a simple discrete region $\mathscr{R}$, then $|\mathrm{f}|$ assumes its absolute maximum on the boundary of $\mathscr{R}$.

Proof: Since $\mathscr{R}$ is finite, $|f|$ assumes an absolute maximum $M$ at a point $z_{1}$ of $\mathscr{R}$. If $z_{1}$ is on the boundary of $\mathscr{R}$, the theorem is proved.

Suppose then that $z_{1}$ is in the interior of $\mathscr{R}$. If $z_{1}$ is even (odd) then Lemma 2.3 enables us to construct an even (odd) chain $C: z_{1}, z_{2}, \ldots, z_{n}$ from $z_{1}$ to an even (odd) boundary point $z_{n}$ of $\mathscr{R}$ with the property that $z_{i}, i=1,2, \ldots, n-1$ is an interior point of $\mathscr{R}$. Let $j$ be the smallest positive integer less than or equal to $n$ for which $\left|f\left(z_{j}\right)\right|<M$. Since $j$ must be greater than one, we know that $z_{j-1}$ exists and is an interior point of $\mathscr{R}$ with the property that $\left|f\left(z_{j-1}\right)\right|=M$. The lattice $\mathscr{L}_{h}$ can clearly be rotated so that $z_{j-1}$ and $z_{j}$ have the orientation shown in figure 3.3.

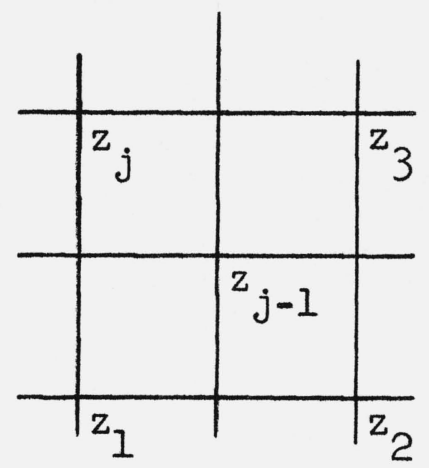

Figure 3.3. An interior point on an even (odd) chain.

Definition 3.3 shows that

$$
f\left(z_{j-1}\right)=\frac{f\left(z_{j}\right)+f\left(z_{1}\right)+f\left(z_{2}\right)+f\left(z_{3}\right)}{4} .
$$

Hence

$$
\left|f\left(z_{j-1}\right)\right| \leqslant \frac{\left|f\left(z_{j}\right)\right|+\left|f\left(z_{1}\right)\right|+\left|f\left(z_{2}\right)\right|+\left|f\left(z_{3}\right)\right|}{4} .
$$

Since

$$
\left|f\left(z_{j}\right)\right|<\left|f\left(z_{j-1}\right)\right|=M,
$$


we see that

$$
f\left(z_{1}\right)+f\left(z_{3}\right)+f\left(z_{5}\right)+f\left(z_{7}\right)-4 f\left(z_{j}\right)=0 . \quad \text { q.e.d. }
$$

$$
M<\frac{M+M+M+M}{4}=M,
$$

which is a contradiction. Hence $\left|f\left(z_{j}\right)\right|=M$ for $j=1$, . . ., $n$ and thus $\left|f\left(z_{n}\right)\right|=M$. This completes the proof of Theorem 3.2.

We can use the preceding theorem to discuss the "Dirichlet problem" for the lattice $\mathscr{L}_{h}$.

THEOREM 3.3: Suppose $\mathscr{R}$ is a simple discrete region containing $\mathrm{i}$ interior points along with $\mathrm{b}$ boundary points. If $\left\{\mathrm{B}_{1}, \mathrm{~B}_{2}, \ldots, \mathrm{B}_{\mathrm{b}}\right\}$ is a set of $\mathrm{b}$ complex numbers, then there is a unique lattice function $\mathrm{f}$ that is discrete harmonic in $\mathscr{R}$ and assumes the values $\mathrm{B}_{1}$, $\mathrm{B}_{2}, \ldots, \mathrm{B}_{\mathrm{b}}$ on the boundary of $\mathscr{R}$.

Proof: Definition 3.3 provides us with a system of $i$ linear equations in $i$ unknowns, which must be satisfied by a discrete harmonic function defined on $\mathscr{R}$. The corresponding homogeneous system is obtained by setting each boundary value of $f$ equal to zero. Theorem 3.2 shows that the homogeneous system has only the trivial solution. Hence, the inhomogeneous system has a unique solution, which proves the theorem.

The next theorem, which is taken from [3], relates the concept of discrete harmonicity to the concept of discrete analyticity.

TheORem 3.4: If $\mathrm{f}$ is a lattice function that is discrete analytic at each square making up a discrete region $\mathscr{R}$, then $\mathrm{f}$ along with its real and imaginary parts is discrete harmonic at each interior point of $\mathscr{R}$.

Proof: Suppose $z_{j}$ is an interior point of $\mathscr{R}$. Then, according to Lemma 2.1, the squares $S_{1}, S_{2}, S_{3}$, and $S_{4}$ shown in figure 3.4 belong to the family of squares used in the construction of $\mathscr{R}$. Hence

$$
\begin{aligned}
& f\left(z_{1}\right)+i f\left(z_{2}\right)+i^{2} f\left(z_{j}\right)+i^{3} f\left(z_{8}\right)=0 \\
& f\left(z_{2}\right)+i f\left(z_{3}\right)+i^{2} f\left(z_{4}\right)+i^{3} f\left(z_{j}\right)=0 \\
& f\left(z_{j}\right)+i f\left(z_{4}\right)+i^{2} f\left(z_{5}\right)+i^{3} f\left(z_{6}\right)=0 \\
& f\left(z_{8}\right)+i f\left(z_{j}\right)+i^{2} f\left(z_{6}\right)+i^{3} f\left(z_{7}\right)=0 .
\end{aligned}
$$

Multiplying the first equation by +1 , the second equation by $-i$, the third equation by -1 , and the fourth equation by $+i$, and then adding the results shows that

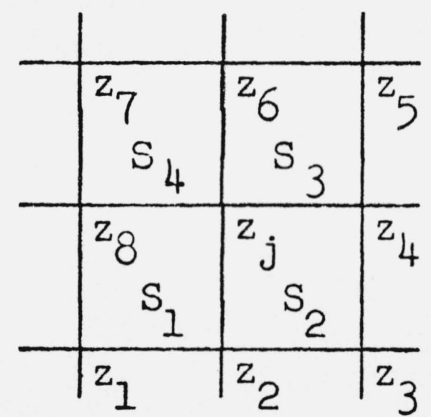

FigURE 3.4. An interior point of a discrete region.
The preceding theory can be used to discuss the existence and uniqueness of a lattice function $f$ that is defined on a simple discrete region $\mathscr{R}$ and has prescribed residue at each square making up $\mathscr{R}$. The following existence and uniqueness theorem is the main result of this paper.

THEOREM 3.5: Let $\mathscr{R}$ be a simple discrete region constructed from $\mathrm{s}$ squares, and suppose that $\mathscr{R}$ has $\mathrm{p}$ lattice points and $\mathrm{b}$ boundary points. If

(i) $\mathrm{z}^{0}$ and $\mathrm{z}^{\mathrm{e}}$ are odd and even lattice points respectively belonging to the discrete region $\mathscr{R}$,

(ii) $\left\{\mathrm{v}^{0}, \mathrm{v}^{\mathrm{e}}\right\}$ is a set of two real numbers,

(iii) $\left\{\mathrm{u}^{\mathrm{j}}\right\}_{\mathrm{j}=1}^{\mathrm{b}}$ is a set of $\mathrm{b}$ real numbers, and

(iv) $\left\{\mathrm{a}^{\mathrm{j}}+\mathrm{ib}^{\mathrm{j}}\right\}_{\mathrm{j}=1}^{\mathrm{s}}$ is a set of $\mathrm{s}$ complex numbers, then there is a unique complex function $\mathrm{f}=\mathrm{u}+\mathrm{iv}$ that. is defined on $\mathscr{R}$ and has the following properties:

(I) $\mathrm{v}$ assumes the values $\mathrm{v}^{0}$ and $\mathrm{v}^{\mathrm{e}}$ at $\mathrm{z}^{0}$ and $\mathrm{z}^{\mathrm{e}}$, respectively.

(II) $\mathrm{u}$ assumes the value $\mathrm{u}^{\mathrm{j}}$ at the $\mathrm{jth}$ boundary point of $\mathscr{R}$.

(III) $\mathrm{L}\left(\mathrm{f}, \mathrm{S}^{\mathrm{j}}\right)=\mathrm{a}^{\mathrm{j}}+\mathrm{i} \mathrm{b}^{\mathrm{j}}$ for $\mathrm{j}=1, \ldots$, s where $\left\{\mathrm{S}^{\mathrm{j}}\right\}_{\mathrm{j}=1}^{\mathrm{s}}$ is the set of squares used in the construction of $\mathscr{R}$.

Proof: Equating the real and imaginary parts of the equation $L\left(f, S^{j}\right)=a^{j}+i b^{j}$ shows that $f=u+i v$ has residue $a^{j}+i b^{j}$ at $S^{j}$ if, and only if,

$$
u\left(z_{2}\right)-u\left(z_{0}\right)=v\left(z_{3}\right)-v\left(z_{1}\right)-a^{j}
$$

and

$$
v\left(z_{2}\right)-v\left(z_{0}\right)=-\left[u\left(z_{3}\right)-u\left(z_{1}\right)+b^{j}\right],
$$

where $z_{0}, z_{1}, z_{2}$, and $z_{3}$ have the orientation shown in figure 3.1. Since $\mathscr{R}$ is constructed from $s$ squares, we obtain a system of $2 s$ linear equations that must be satisfied by the real and imaginary parts of the function $f$ whose prescribed residues are $\left\{a^{j}+i b^{j}\right\}_{j=1}^{s}$. There are $2 p-b-2$ unknowns because $u$ is given at $b$ boundary points and $v$ is specified at $z^{0}$ and $z^{e}$. Lemma 2.5 shows that the number of unknowns is equal to the number of equations in the system. The corresponding homogeneous system is obtained by setting $u^{1}, u^{2}, . . ., u^{b}, v^{0}, v^{e}, a^{1}, \ldots ., a^{s}, b^{1}, \ldots$, $b^{s-1}$, and $b^{s}$ equal to zero.

Suppose $\bar{u}$ and $\bar{v}$ represent a solution of the homogeneous system. Then $\bar{f} \equiv \bar{u}+i \bar{v}$ is discrete analytic on $\mathscr{R}$, and Theorem 3.4 shows that $\bar{u}$ is discrete harmonic at interior points of $\mathscr{R}$. We conclude from Theorem 3.3 that $\bar{u}$ is identically zero. Now suppose that $z^{0}$ is a vertex of a square $S_{1}$ and that $\left\{S_{j}\right\}_{j=1}^{s}$ is the corresponding sequence of squares given by Lemma 2.4 . Since $\bar{u}$ is identically zero and $\bar{v}\left(z^{0}\right)=0$, the "Cauchy Riemann equations" of Theorem 3.1 show that $\bar{v}$ is zero at the other odd lattice point of $S_{1}$. Using the second conclusion of Lemma 2.4, we observe that . $\bar{v}$ is, a fortiori, zero at one of the odd vertices of $S_{2}$ and hence zero at the other odd vertex of $S_{2 \text {. }}$ Repeating 
this argument an additional $(s-2)$ times, we conclude that $\bar{v}$ is zero at all odd lattice points of $\mathscr{R}$. The same argument with $z^{0}$ replaced by $z^{e}$ and the word odd replaced by the word even shows that $\bar{v}$ is zero at all even lattice points of $\mathscr{R}$. Hence, both $\bar{u}$ and $\bar{v}$ are identically zero on $\mathscr{R}$ and thus the homogeneous system has only the trivial solution. This means the inhomogeneous system has a unique solution, which proves the theorem.

COROLlaRY 3.1: If u is a real discrete harmonic function that is defined on a simple discrete region $\mathscr{R}$, then $\mathrm{u}$ is the real part of a lattice function $\mathrm{u}+\mathrm{iv}$ that is discrete analytic on $\mathscr{R}$.

Proof: Theorem 3.5 shows the existence of a discrete analytic function $\bar{u}+i v$ with $\bar{u}$ assuming the boundary values taken on by $u$. Then Theorem 3.3 shows that $\bar{u}=u$.

The real function $v$ of Corollary 3.1 is called a dis. crete harmonic conjugate of $u$. In the theory of functions of a continuous complex variable a particular harmonic conjugate of a real harmonic function is uniquely determined by its value at a given point of its domain. An inspection of Theorem 3.5 shows that a particular discrete harmonic conjugate $v$ of a discrete harmonic function $u$ is uniquely determined by its values at two different points $z^{0}$ and $z^{e}$ of its domain.
The difference between the two theories is due to the fact that the discrete harmonic operator $H$ does not relate a discrete function restricted to the odd lattice to the discrete function restricted to the even lattice. In solving the Dirichlet problem for $\mathscr{L}_{h}$, we must, in a sense, solve two Dirichlet problems, one for the even lattice and one for the odd lattice.

\section{References}

[1] Isaacs, R. P., A finite difference function theory, Universidad Nacional Tucuman Revista 2, 177-201 (1941).

[2] Ferrand, J., Fonctions preharmonique et fonctions preholomorphis, 2d series, Bull. Sci. Math. 68, 152-180 (1944).

[3] Duffin, R. J., Basic properties of discrete analytic functions, Duke Math. Journal 23, 335-363 (1956).

[4] Duffin, R. J., and C. Duris, A convolution product for discrete function theory, Duke Math. Journal 31, 199-220 (1964).

[5] Duffin, R. J., and C. Duris, Discrete analytic continuation of solutions of difference equations, J. Math. Anal. and Appl., 9, 252-267 (1964).

[6] Duffin, R. J., and Elmor L. Peterson, The Discrete analogue of a class of entire functions, to appear in J. Math. Anal. and Appl., 1967.

[7] Washburn, A., D transform in discrete function theory, Ph. D. thesis in mathematics, Carnegie Institute of Technology, 1965.

[8] Berge, C., The Theory of Graphs and its Applications (John Wiley \& Sons Inc., New York, N.Y., 1962).

(Paper 71B2 \& 3-205) 